\title{
Síndrome de Burnout en docentes de Latinoamérica: Una revisión sistemática
}

\author{
Burnout syndrome in teachers from Latin America: A systematic review
}

Yuranny Alejandra Tabares-Díaz ${ }^{1 *}$ orcid.org/0000-0002-1717-6710

Viviana Alexandra Martínez-Daza ${ }^{1}$ orcid.org/0000-0001-5951-5250

Sonia Maritza Matabanchoy-Tulcán ${ }^{1}$ orcid.org/0000-0002-3681-8440

1 Grupo de Investigación CONEPSI. Departamento de Psicología. Universidad de Nariño, Pasto, Colombia.

\section{Resumen}

Introducción: El Síndrome de Burnout (SB) se entiende como un fenómeno que incluye cansancio emocional, despersonalización y reducida realización personal, generando consecuencias multivariadas a nivel físico, psicológico, social y laboral. Objetivo: Identificar la producción bibliográfica en Latinoamérica sobre el SB en docentes en el período comprendido entre los años 2008 a 2018. Materiales y métodos: Revisión sistemática de literatura basada en las directrices PRISMA-P. Se realizó un rastreo bibliográfico de publicaciones en las bases de datos Web of Science, Scopus, Directory of Open Access Journals y EBSCOhost, utilizando las palabras clave: Burnout AND docentes y sus expresiones equivalentes en inglés. Resultados: Se seleccionaron un total de 53 artículos, evidenciando una mayor proporción de publicaciones en Brasil, en los años 2017, 2014 y 2018, en muestras mayoritariamente de docentes de instituciones de educación básica, con predominio de estudios cuantitativos centrados en el análisis de variables relacionadas al SB. Conclusiones: Las investigaciones sobre SB se focalizan sólo en algunos países latinoamericanos, con alcances paradigmáticos y metodológicos específicos, señalando la necesidad de realizar estudios primarios sobre dicho fenómeno.

Palabras clave: Revisión sistemática; agotamiento psicológico; docentes; Latinoamérica (Fuente: DeCS, Bireme).

\begin{abstract}
Introduction: The Burnout Syndrome (BS) is understood as a phenomenon that includes emotional fatigue, depersonalization, and reduced personal accomplishment, which have multivariate consequences at physical, psychological, social, and occupational levels. Objective: To examine Latin American reports on BS in teachers that were published between 2008 and 2018. Materials and methods: A systematic literature search was conducted, which was based on the PRISMA-P guidelines and included the Web of Science, Scopus, Directory of Open Access Journals and EBSCOhost databases. The keywords used were: "Burnout" AND "teachers", together with the corresponding translation in English. Results: A total of 53 articles were selected, which were mostly published in Brazil in 2014, 2017, and 2018. The majority of reports were on teachers from basic education institutions and corresponded to quantitative studies focused on the analysis of variables related to BS. Conclusions: Latin American research on BS is mostly concentrated in some Latin American countries and has specific paradigmatic and methodological scopes. The need to carry out primary studies about this phenomenon is highlighted.
\end{abstract}

Keywords: Systematic review; burnout, psychological; faculty; Latin America (Source: DeCS, Bireme).

\footnotetext{
*Autor de correspondencia Yuranny Alejandra Tabares Díaz e-mail: alejataba15@hotmail.com
} 


\section{Introducción}

En las últimas décadas, las transformaciones en el mundo laboral derivadas de los cambios tecnológicos, organizacionales y gerenciales de las empresas, las nuevas exigencias y requisitos impuestos en el trabajo(1), han propiciado en conjunto la aparición de problemáticas psicosociales, entre ellas el Síndrome de Burnout (SB), catalogado por la Red Mundial de Salud Ocupacional (2) como un factor de riesgo laboral que incide negativamente en la salud, seguridad, bienestar de las personas y calidad de vida en el trabajo(3). De este modo, en el contexto laboral, la docencia es considerada como una de las profesiones más vulnerables a desarrollar problemas de salud mental, entre los que se incluye el Burnout( ${ }^{(4)}$.

Desde la etiología, tal como lo expone Jorquera et al.(5) este síndrome comenzó a investigarse en los años 70 por autores como Freudenberger ${ }^{(6)}$ para hacer alusión al desgaste causado en un profesional, debido al exceso de fuerza y/o demandas de energía dispuestas para alcanzar las metas laborales; más adelante, Maslach et al.(7), lo conceptualizaron como un síndrome psicológico de agotamiento emocional, despersonalización y reducida realización personal, en individuos que trabajan con otras personas; en la última década, Gil-Monte ${ }^{(8,9)}$ lo definió como una respuesta psicológica al estrés laboral crónico, caracterizado por un deterioro físico, cognitivo, afectivo, emocional e interpersonal.

Lo expuesto, ha dado origen a diversos modelos explicativos ${ }^{(10)}$, sin lograr consenso en una definición conceptual globalmente aprobada, ni tampoco su operacionalización, ocasionando críticas y reflexiones en torno a ello(11).

Una de las clasificaciones más empleadas es la de GilMonte (9), que categoriza los modelos en cuatro grupos. El primero corresponde a los modelos de la teoría cognoscitiva del yo, los cuales señalan la influencia de la cognición en las percepciones y comportamientos de los individuos(12); se destacan los modelos de Harrison(13), Pines ${ }^{(14)}$ y Cherniss ${ }^{(15)}$. El segundo grupo, hace referencia a los modelos basados en las teorías de intercambio social, que atribuyen la etiología del SB a la percepción de ausencia de equidad o ganancia del individuo, al compararse con otros trabajadores ${ }^{(10)}$; se integran los modelos de Buunk et al.(16) y Hobfoll et al.(17).

En el tercer grupo, los modelos desde la teoría organizacional enfatizan que el SB surge como respuesta de las disfunciones existentes en el rol del trabajo, el clima laboral, la cultura o la estructura organizacional(5); se incluyen los modelos de Golembiewski et al.(18), Cox et al.(19) y Winnubst ${ }^{(20)}$. Por último, los modelos de la teoría estructural, atribuyen el origen del SB al desequilibrio entre la percepción de las demandas y la capacidad de respuesta del individuo, sumado a los antecedentes personales, interpersonales y organizacionales implicados, incorporando el modelo de Gil-Monte et al.(21).

Es importante mencionar que existen diferentes variables individuales como el sexo, edad, estado civil, nivel de estudios, composición familiar, habilidades sociales, estilos de afrontamiento frente al estrés, tolerancia al fracaso y a la frustración, entre otras ${ }^{10,22-}$ 25); y variables sociales, intralaborales y extralaborales, incluyendo escaso apoyo social, multiplicidad de tareas, espacios inadecuados, falta de tiempo y remuneración insuficiente, clima organizacional y relaciones sociales laborales inadecuadas, etc., las cuales constituyen factores de riesgo que incrementan la probabilidad del sujeto de

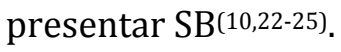

Adicionalmente, se destaca el carácter multivariado de las consecuencias del SB, las cuales generan perjuicios en las condiciones de vida del individuo a nivel físico, psicosocial y laboral, incluyendo conflictos familiares y profesionales, problemas psicosomáticos, afecciones cardiovasculares, gastrointestinales, musculares y respiratorias, alteraciones en el estado emocional, deterioro de la salud psicológica, agotamiento mental, percepción negativa del estado de salud en general, abuso de sustancias, disminución en la productividad del trabajo, aumento del ausentismo y bajo umbral para soportar la presión, dentro de las principales(26-28).

En relación a las publicaciones científicas latinoamericanas, múltiples investigaciones sobre el SB se han orientado a la delimitación del concepto, generación de modelos explicativos, evaluación y diagnóstico y en menor grado al planteamiento de estrategias de prevención e intervención; se reconoce también, la heterogeneidad en la operacionalización del constructo, los instrumentos de evaluación y el amplio énfasis de estudio del SB en personal del área de la salud, en comparación a profesionales de contextos educativos $(29,30)$. 
Por lo anterior, se destaca la importancia de continuar con procesos de investigación científica sobre el SB en población docente, al ser uno de los conjuntos gremiales en los que la naturaleza de su profesión es una condición que puede influir en el desarrollo de estrés laboral, aspecto que a su vez incide negativamente en los procesos de enseñanza, calidad educativa y relaciones interpersonales(22,28), con una prevalencia entre el $4,2 \%$ a $16 \%$ en niveles severos y del $42 \%$ a $60 \%$ en niveles altos, en al menos una de las dimensiones evaluadas del fenómeno(23,31). Salgado et al.(32) y Olaya(33) identificaron que dependiendo de la etapa educativa en la que laboran los docentes, al menos el $40 \%$ de los evaluados tienen $\mathrm{SB}$, siendo preponderantemente mayor la prevalencia y riesgo de presentación del síndrome en docentes de instituciones de educación secundaria en comparación a aquellos de enseñanza primaria(32,33).

Por otro lado, los índices de SB en sus diferentes dimensiones en docentes de instituciones de educación superior, varían entre el $5 \%$ a $24(24,34)$. A partir de lo anterior, se vio la necesidad de desarrollar esta revisión sistemática que tuvo por objetivo identificar la producción bibliográfica en Latinoamérica sobre el SB en docentes en el período comprendido entre los años 2008 a 2018.

\section{Materiales y métodos}

\section{Unidad de análisis}

Se analizaron 53 artículos que reportaban resultados sobre SB en docentes en Latinoamérica.

\section{Tipo de estudio}

Se llevó a cabo una revisión sistemática de literatura, dado que permite condensar los resultados de un acervo de estudios, presentando una descripción a manera de síntesis cualitativa de la información encontrada, a fin de dar respuesta a una pregunta de investigación específica(35).

\section{Instrumentos}

Se realizó una matriz de registro documental basada en las directrices del PRISMA-P (Preferred Reporting Items for Systematic Review and Meta-Analysis Protocols), que proporciona un conjunto mínimo de ítems para la inclusión de artículos, con el objetivo de orientar la preparación del protocolo de búsqueda, la planificación y el análisis de la revisión. Dicha matriz incorporó los siguientes criterios: título, autores, año de publicación, idioma, país del estudio, nombre de la revista y base de datos, resumen, número y tipo de muestra, objetivo(s), tipo de investigación, tipo y nombres de instrumentos de medida y técnicas utilizadas, propiedades psicométricas informadas, $\mathrm{y}$ principales resultados de los estudios.

\section{Diseño y procedimiento}

Para el desarrollo de la revisión sistemática se realizaron las siguientes etapas: 1) formulación de la pregunta y objetivo de investigación, 2) especificación de los criterios de inclusión de los artículos, 3) formulación del plan de búsqueda de literatura, 4) búsqueda de artículos en las bases de datos seleccionadas, 5) evaluación de la calidad de los artículos de acuerdo a criterios establecidos, 6) sistematización de la información y 7) interpretación y presentación de resultados.

El rastreo bibliográfico consideró publicaciones latinoamericanas sobre SB en docentes, en las bases de datos Web of Science (WOS), Scopus, Directory of Open Access Journals (DOAJ) y EBSCOhost, seleccionadas por ser algunas de las más reconocidas y multidisciplinarias a nivel internacional, además de la disponibilidad de acceso bases pagadas (WOS, Scopus y EBSCOhost). Los términos de búsqueda empleados en las bases de datos correspondieron a: Burnout $A N D$ docentes, Burnout AND profesores y Burnout AND teachers. Los resultados fueron probados por cada país latinoamericano y en todos los idiomas disponibles, con el fin de recoger todas las opciones posibles sin obviar información.

La búsqueda de artículos en la base de datos WOS se ejecutó en el apartado de temas, en Scopus en títulos, resúmenes y palabras clave, en EBSCOhost en documentos de texto completo y en $D O A J$ con el filtro de búsqueda total; lo anterior, con el propósito de minimizar el sesgo y cotejar todos los artículos.

Los criterios de inclusión para la revisión fueron: a) estudios con tema central o relación explícita en SB en docentes, b) investigaciones desarrolladas en países latinoamericanos entre los años 2008 a 2018, en idioma español, inglés y/o portugués; c) artículos de texto completo que en sus apartados (título, introducción, objetivos, resultados o discusión) mencionaran el concepto de Burnout en docentes o su expresión equivalente en español.

Se descartaron artículos duplicados en las bases de datos, tomando sólo uno de ellos. Se excluyeron aquellos estudios cuyo eje central de investigación no correspondía a $\mathrm{SB}$, su contexto de trabajo no era la 
docencia, su carácter no era empírico y sus resultados eran preliminares.
De esta manera, se concretó el desarrollo de cuatro fases de revisión, procedimiento propuesto por la declaración PRISMA (Figura 1).

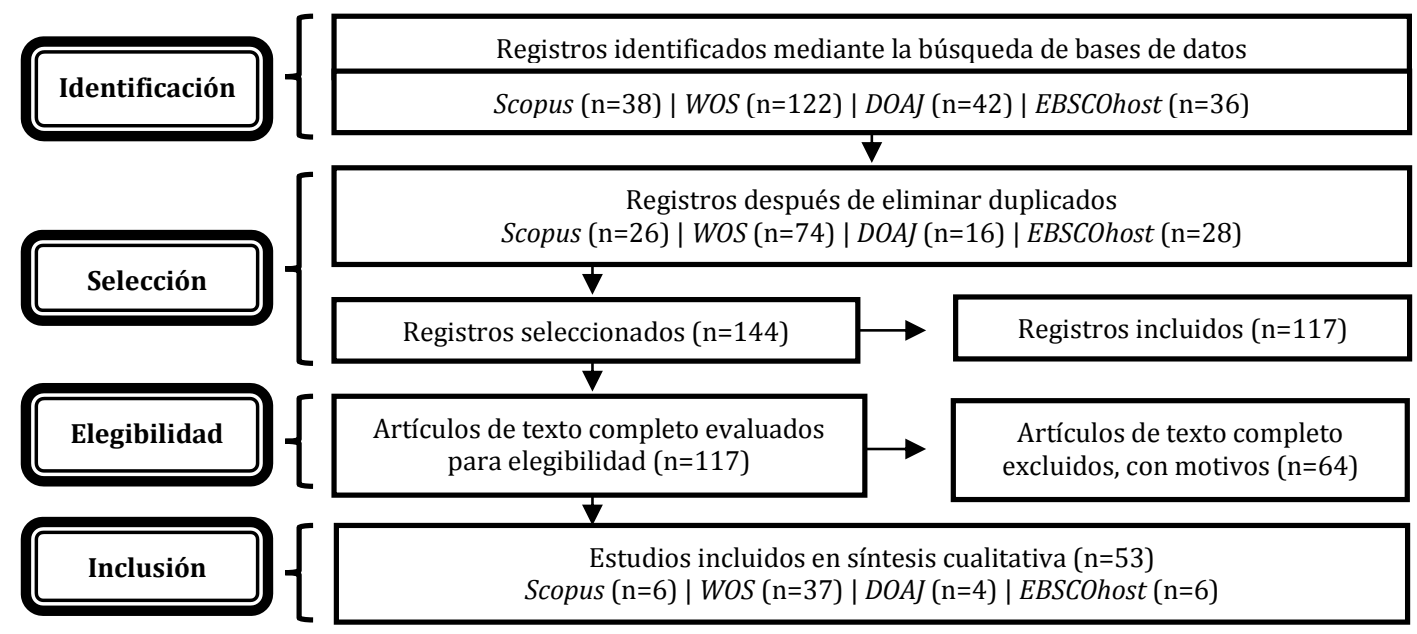

Figura 1. Flujo de información a través de las diferentes fases de una revisión sistemática

\section{Resultados}

Se identificaron 53 artículos que cumplieron los criterios de inclusión. Los resultados de los estudios se organizaron en tres categorías: a) lugar, año e idioma de publicación, b) alcances metodológicos y c) objetivos y resultados.

\section{Lugar, año e idioma de publicación}

Brasil presentó la mayor producción científica respecto del objeto de estudio $(n=31)$, seguido de México (n=7), Colombia $(n=6)$ y Chile $(n=5)$. Los años 2017 y 2014 fueron los de mayor publicación con 8 artículos cada uno, seguidos del año 2018 con 7 artículos, equivalentes a un $43,4 \%$ del total analizados. Frente al idioma de publicación, 26 artículos están en portugués, 22 en español y 5 en inglés. En la Tabla 1 se presentan los principales datos de los artículos que forman parte de esta revisión.

\section{Alcance metodológico de los estudios}

Tipos de investigación: El 77,4\% de los 53 artículos revisados correspondieron a estudios cuantitativos, $9,4 \%$ a mixtos, $7,5 \%$ a revisiones sistemáticas y $5,7 \%$ a cualitativos.

Tabla 1. Caracterización de los estudios incluidos en la revisión sistemática

\begin{tabular}{|c|c|c|c|c|}
\hline 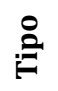 & Cita & $\begin{array}{l}\text { País e } \\
\text { idioma }\end{array}$ & Objetivo & Objetivo \\
\hline \multirow{3}{*}{ 莡 } & Diehl y Carlotto, $2014^{36}$ & $\begin{array}{l}\text { Brasil } \\
\text { Inglés }\end{array}$ & $\begin{array}{l}\text { Explorar el conocimiento de los profesores sobre el SB y comprender los } \\
\text { elementos que se utilizan para su interpretación. }\end{array}$ & \multirow{3}{*}{ DCF } \\
\hline & $\begin{array}{l}\text { Gomes, Santos y Medeiros, } \\
2010^{37}\end{array}$ & $\begin{array}{l}\text { Brasil } \\
\text { Portugués }\end{array}$ & $\begin{array}{l}\text { Investigar la clínica sugerente de Burnout en docentes de escuelas } \\
\text { primarias públicas y reflexionar sobre el trabajo de enfermería. }\end{array}$ & \\
\hline & $\begin{array}{l}\text { Esteves, Santos y Rigolon, } \\
2014^{38}\end{array}$ & $\begin{array}{l}\text { Brasil } \\
\text { Portugués }\end{array}$ & $\begin{array}{l}\text { Evaluar la presencia de síntomas de SB en profesionales de escuelas } \\
\text { públicas y privadas e identificar qué grupo estaría más propenso a } \\
\text { desarrollarla. }\end{array}$ & \\
\hline \multirow{3}{*}{ 莣 } & Ilaja y Reyes, $2016^{39}$ & $\begin{array}{l}\text { Ecuador } \\
\text { Español }\end{array}$ & $\begin{array}{l}\text { Explorar los aspectos relacionados con el SB en las tareas realizadas por } \\
\text { los docentes universitarios y sus variables mediadoras. }\end{array}$ & \multirow{3}{*}{ AVR } \\
\hline & $\begin{array}{l}\text { Jiménez, Jara y Miranda, } \\
2012^{31}\end{array}$ & $\begin{array}{l}\text { Chile } \\
\text { Español }\end{array}$ & $\begin{array}{l}\text { Analizar la relación entre las variables SB, apoyo social y satisfacción } \\
\text { laboral. }\end{array}$ & \\
\hline & $\begin{array}{l}\text { Mazon, Carlotto, y Câmara, } \\
2008^{40}\end{array}$ & $\begin{array}{l}\text { Brasil } \\
\text { Portugués }\end{array}$ & $\begin{array}{l}\text { Verificar la existencia de asociación entre las dimensiones del SB y } \\
\text { estrategias de enfrentamiento en profesores de escuelas municipales de } \\
\text { Brasil. }\end{array}$ & \\
\hline
\end{tabular}




\begin{tabular}{|c|c|c|}
\hline $\begin{array}{l}\text { Silva, Bolsoni, Rodrigues y } \\
\text { Capellini, } 2015^{25}\end{array}$ & $\begin{array}{l}\text { Brasil } \\
\text { Portugués }\end{array}$ & $\begin{array}{l}\text { Correlacionar y predecir las variables condiciones de trabajo del profesor, } \\
\text { indicadores de SB, prácticas educativas del profesor y repertorio de } \\
\text { habilidades sociales y problemas de comportamiento de los alumnos. }\end{array}$ \\
\hline Costa y Selete, $2012^{41}$ & $\begin{array}{l}\text { Brasil } \\
\text { Inglés }\end{array}$ & $\begin{array}{l}\text { Analizar el ambiente de trabajo que afecta la calidad de vida para maestros } \\
\text { de escuelas públicas. }\end{array}$ \\
\hline $\begin{array}{l}\text { Santana, Marchi, Junior, } \\
\text { Girondoli y Chiappeta, } \\
2012^{42}\end{array}$ & $\begin{array}{l}\text { Brasil } \\
\text { Inglés }\end{array}$ & $\begin{array}{l}\text { Evaluar la aparición del SB en } 100 \text { docentes de seis altos públicos, las } \\
\text { escuelas y su correlación con las condiciones de trabajo en Brasil. }\end{array}$ \\
\hline $\begin{array}{l}\text { Bedoya, Vega, Severiche y } \\
\text { Meza, } 2017^{43}\end{array}$ & $\begin{array}{l}\text { Colombia } \\
\text { Español }\end{array}$ & $\begin{array}{l}\text { Evaluar el síndrome de quemado (burnout) y factores asociados en } \\
\text { docentes de una institución superior del caribe colombiano. }\end{array}$ \\
\hline $\begin{array}{l}\text { Ruiz, Pando, Aranda y } \\
\text { Almeida, 201444 }\end{array}$ & $\begin{array}{l}\text { México } \\
\text { Español }\end{array}$ & $\begin{array}{l}\text { Determinar la relación que existe entre el SB y el Work Engagement con } \\
\text { variables demográficas y laborales en docentes universitarios de } \\
\text { Zacatecas. }\end{array}$ \\
\hline $\begin{array}{l}\text { Moreno, Corso, Sanz, } \\
\text { Rodriguez y Boada, } 2010^{45}\end{array}$ & $\begin{array}{l}\text { Perú } \\
\text { Español }\end{array}$ & $\begin{array}{l}\text { Presentar una aplicación del modelo de Demandas-Recursos Laborales, } \\
\text { sus relaciones con el burnout y el engagement y sus efectos sobre la } \\
\text { sintomatología y la satisfacción vital. }\end{array}$ \\
\hline $\begin{array}{l}\text { Jorquera, Orellana, Tapia y } \\
\text { Vergara, } 2014^{5}\end{array}$ & $\begin{array}{l}\text { Chile } \\
\text { Español }\end{array}$ & $\begin{array}{l}\text { Establecer la relación entre burnout y una serie de variables } \\
\text { sociodemográficas y organizacionales. }\end{array}$ \\
\hline Salgado y Leria, $2018^{32}$ & $\begin{array}{l}\text { Chile } \\
\text { Español }\end{array}$ & $\begin{array}{l}\text { Analizar los niveles del SB y la percepción de calidad de vida profesional } \\
\text { en función de los estilos de personalidad y variables sociodemográficas y } \\
\text { sociolaborales. }\end{array}$ \\
\hline $\begin{array}{l}\text { Souza, Tenório, Cruz, Sousa } \\
\text { y Gomes, } 2016^{46}\end{array}$ & $\begin{array}{l}\text { Brasil } \\
\text { Portugués }\end{array}$ & $\begin{array}{l}\text { Verificar la relación entre las dimensiones del SB y los valores humanos de } \\
\text { los profesores de la red pública estatal de Brasil. }\end{array}$ \\
\hline Marenco y Ávila, $2016^{22}$ & $\begin{array}{l}\text { Colombia } \\
\text { Español }\end{array}$ & $\begin{array}{l}\text { Evaluar la existencia de diferencias entre las manifestaciones de burnouty } \\
\text { los problemas de salud mental en docentes de acuerdo con sus } \\
\text { características demográficas y sociolaborales. }\end{array}$ \\
\hline $\begin{array}{l}\text { Rodríguez y Sánchez, } \\
2018^{4}\end{array}$ & $\begin{array}{l}\text { Perú } \\
\text { Español }\end{array}$ & $\begin{array}{l}\text { Determinar las diferencias entre los componentes del SB en docentes de } \\
\text { una universidad privada de Lima según variables sociodemográficas. }\end{array}$ \\
\hline $\begin{array}{l}\text { Bruch, Aerts, Guimarães y } \\
\text { Gonçalves, } 2016^{47}\end{array}$ & $\begin{array}{l}\text { Brasil } \\
\text { Portugués }\end{array}$ & $\begin{array}{l}\text { Estudiar el SB en docentes de las instituciones privadas de enseñanza } \\
\text { superior en Brasil y su relación con características demográficas, laborales } \\
\text { y estilo de vida. }\end{array}$ \\
\hline Sánchez y Velasco, $2017^{48}$ & $\begin{array}{l}\text { México } \\
\text { Español }\end{array}$ & $\begin{array}{l}\text { Describir y estimar la comorbilidad del SB, depresión y ansiedad en una } \\
\text { muestra de profesores de educación básica de México. }\end{array}$ \\
\hline $\begin{array}{l}\text { Tabeleão, Tomasi y Neves, } \\
2011^{49}\end{array}$ & $\begin{array}{l}\text { Brasil } \\
\text { Portugués }\end{array}$ & $\begin{array}{l}\text { Investigar los niveles de calidad de vida en maestros de escuelas estatales } \\
\text { y municipales de primaria y secundaria y relacionarlos con características } \\
\text { sociodemográficas y de procesos de trabajo. }\end{array}$ \\
\hline Carlotto y Câmara, 201750 & $\begin{array}{l}\text { Brasil } \\
\text { Inglés }\end{array}$ & $\begin{array}{l}\text { Evaluar tres perfiles discriminantes de SB en profesores, de acuerdo con } \\
\text { factores psicosociales. }\end{array}$ \\
\hline $\begin{array}{l}\text { Camargo, Nesello, Santos, } \\
\text { Lopes, Durán, Eumann et } \\
\text { al., } 2015^{51}\end{array}$ & $\begin{array}{l}\text { Brasil } \\
\text { Portugués }\end{array}$ & $\begin{array}{l}\text { Identificar factores asociados con los peores niveles en las tres } \\
\text { dimensiones de Burnout en maestros de educación básica en Brasil. }\end{array}$ \\
\hline $\begin{array}{l}\text { Carlotto, Silva, Vidal y } \\
\text { Diehl, 201552 }\end{array}$ & $\begin{array}{l}\text { Brasil } \\
\text { Portugués }\end{array}$ & $\begin{array}{l}\text { Evaluar si la autoeficacia funciona como variable mediadora en la relación } \\
\text { entre la sobrecarga de trabajo y las dimensiones de burnout. }\end{array}$ \\
\hline $\begin{array}{l}\text { García, Escorcia y Perez, } \\
2017^{53}\end{array}$ & $\begin{array}{l}\text { Colombia } \\
\text { Español }\end{array}$ & $\begin{array}{l}\text { Analizar la relación del SB y los sentimientos de autoeficacia con el } \\
\text { rendimiento académico en profesores de una universidad privada. }\end{array}$ \\
\hline $\begin{array}{l}\text { Suda, Coelho, } \quad \text { Bertaci } \quad y \\
\text { Santos, } 2011^{54}\end{array}$ & $\begin{array}{l}\text { Brasil } \\
\text { Portugués }\end{array}$ & $\begin{array}{l}\text { Verificar la relación entre nivel de salud general, dolor y frecuencia de } \\
\text { síntomas musculoesqueléticos y SB en profesores universitarios. }\end{array}$ \\
\hline $\begin{array}{l}\text { Cárdenas, Méndez } \quad \text { y } \\
\text { González, 201455 }\end{array}$ & $\begin{array}{l}\text { México } \\
\text { Español }\end{array}$ & $\begin{array}{l}\text { Examinar la relación entre los puntajes obtenidos por docentes } \\
\text { universitarios en la evaluación de su desempeño con el grado de estrés } \\
\text { percibido y con la presencia de indicadores del SB. }\end{array}$ \\
\hline $\begin{array}{l}\text { Dalagasperina y Monteiro, } \\
201456\end{array}$ & $\begin{array}{l}\text { Brasil } \\
\text { Portugués }\end{array}$ & $\begin{array}{l}\text { Analizar los factores de estrés en el trabajo y las variables } \\
\text { sociodemográficas que predicen las dimensiones del SB en maestros de } \\
\text { primaria de Brasil. }\end{array}$ \\
\hline Carlotto y Câmara, 201757 & $\begin{array}{l}\text { Brasil } \\
\text { Portugués }\end{array}$ & $\begin{array}{l}\text { Evaluar los estresores ocupacionales de naturaleza psicosocial, como } \\
\text { posibles predictores de las dimensiones del SB en una muestra de } \\
\text { profesores universitarios. }\end{array}$ \\
\hline $\begin{array}{l}\text { Chavarría, Colunga, Loria y } \\
\text { Peláez, } 201758\end{array}$ & $\begin{array}{l}\text { México } \\
\text { Español }\end{array}$ & $\begin{array}{l}\text { Identificar la presencia de SB en médicos dedicados a labores docentes y } \\
\text { los factores asociados a su presentación. }\end{array}$ \\
\hline
\end{tabular}


Correa, Muñoz y Chaparro, Colombia $2010^{59} \quad$ Español

Lima, Cardoso, Pereira, Brasil Alves, Gomes y Amaral, Portugués $2018^{60}$

\begin{tabular}{|c|c|c|c|}
\hline Silva y Almeida, $2011^{61}$ & $\begin{array}{l}\text { Brasil } \\
\text { Portugués }\end{array}$ & $\begin{array}{l}\text { Comparar la presencia de indicadores de SB en tres grupos de docentes } \\
\text { que actúan en el primer ciclo de enseñanza fundamental en Brasil. }\end{array}$ & \\
\hline $\begin{array}{l}\text { Díaz, López y Varela, } \\
2012^{62}\end{array}$ & $\begin{array}{l}\text { Colombia } \\
\text { Español }\end{array}$ & $\begin{array}{l}\text { Establecer los factores asociados al SB en docentes de dos instituciones } \\
\text { educativas privada y pública de Colombia. }\end{array}$ & \\
\hline $\begin{array}{l}\text { Machado, Nunes y Absalão, } \\
200963\end{array}$ & $\begin{array}{l}\text { Brasil } \\
\text { Portugués }\end{array}$ & $\begin{array}{l}\text { Evaluar el índice de Burnout en profesores de la red pública de la } \\
\text { enseñanza fundamental en< Brasil. }\end{array}$ & \\
\hline Braun y Carlotto, 201464 & $\begin{array}{l}\text { Brasil } \\
\text { Portugués }\end{array}$ & $\begin{array}{l}\text { Verificar si existen diferencias en índices de dimensiones de SB, perfil } 1 \text { y } \\
2 \text { entre profesores de educación especial y regular. }\end{array}$ & \\
\hline $\begin{array}{l}\text { Costa, Gil-Monte, Possobon } \\
\text { y Ambrosano, } 201365\end{array}$ & $\begin{array}{l}\text { Brasil } \\
\text { Portugués }\end{array}$ & $\begin{array}{l}\text { Investigar la prevalencia del SB en una muestra de profesores } \\
\text { universitarios brasileños. }\end{array}$ & \\
\hline $\begin{array}{l}\text { Silva, Maia, Lima, Guedes, } \\
\text { Pedreira, Silva y Petroski, } \\
201766\end{array}$ & $\begin{array}{l}\text { Brasil } \\
\text { Portugués }\end{array}$ & $\begin{array}{l}\text { Verificar la prevalencia y los factores asociados al síndrome del } \\
\text { agotamiento profesional en los profesores de la red pública de las } \\
\text { enseñanzas infantil, fundamental y media. }\end{array}$ & \\
\hline $\begin{array}{l}\text { Silva, Bolsoni y Loureiro, } \\
2018^{67}\end{array}$ & $\begin{array}{l}\text { Brasil } \\
\text { Portugués }\end{array}$ & $\begin{array}{l}\text { Determinar la prevalencia de agotamiento y depresión en los profesores } \\
\text { de primaria e e investigar las posibles } \\
\text { correlaciones entre el agotamiento, la depresión, demográficos y variables } \\
\text { organizacionales. }\end{array}$ & \multirow{3}{*}{$\begin{array}{l}\text { PCF/ } \\
\text { AVR }\end{array}$} \\
\hline $\begin{array}{l}\text { Batista, Carlotto, Coutinho } \\
\text { y Augusto, } 2010^{68}\end{array}$ & $\begin{array}{l}\text { Brasil } \\
\text { Portugués }\end{array}$ & $\begin{array}{l}\text { Evaluar la prevalencia del SB en profesores de enseñanza primaria de } \\
\text { escuelas municipales de Brasil y su relación con variables } \\
\text { sociodemográficas y laborales. }\end{array}$ & \\
\hline Carlotto, $2011^{69}$ & $\begin{array}{l}\text { Brasil } \\
\text { Portugués }\end{array}$ & $\begin{array}{l}\text { Identificar la prevalencia del SB y su relación con factores } \\
\text { sociodemográficos y ocupacionales en docentes. }\end{array}$ & \\
\hline Dalcin y Carlotto, $2018^{70}$ & $\begin{array}{l}\text { Brasil } \\
\text { Portugués }\end{array}$ & Evaluar el efecto de una intervención para SB en profesores. & PI \\
\hline Calderón, 200971 & $\begin{array}{l}\text { Costa Rica } \\
\text { Español }\end{array}$ & $\begin{array}{l}\text { Validar y realizar el análisis factorial de MBI (versión castellana) en } \\
\text { educadores costarricenses. }\end{array}$ & \multirow{3}{*}{ VP } \\
\hline $\begin{array}{l}\text { Gil-Monte, Unda } \\
\text { Sandoval, } 2009^{72}\end{array}$ & $\begin{array}{l}\text { México } \\
\text { Español }\end{array}$ & Validar el CESQT en maestros mexicanos de educación básica. & \\
\hline $\begin{array}{l}\text { Gil-Monte, Carlotto } \quad \mathrm{y} \\
\text { Câmara, } 2010^{73}\end{array}$ & $\begin{array}{l}\text { Brasil } \\
\text { Inglés }\end{array}$ & $\begin{array}{l}\text { Evaluar la validez factorial y la consistencia interna de la versión brasileña } \\
\text { del SBI. }\end{array}$ & \\
\hline $\begin{array}{l}\text { Preciado, Pozos, Colunga, } \\
\text { Vázquez y Ávalos, } 201774\end{array}$ & $\begin{array}{l}\text { México } \\
\text { Español }\end{array}$ & $\begin{array}{l}\text { Determinar la relación de factores psicosociales con agotamiento } \\
\text { emocional laboral y el SB. }\end{array}$ & \multirow{2}{*}{ AVR } \\
\hline Muñoz y Correa, 201475 & $\begin{array}{l}\text { Colombia } \\
\text { Español }\end{array}$ & $\begin{array}{l}\text { Identificar la relación entre el SB y las prácticas educativas en docentes de } \\
\text { primaria y secundaria. }\end{array}$ & \\
\hline Cuadra, Lee y Sossa, 200976 & $\begin{array}{l}\text { Chile } \\
\text { Español }\end{array}$ & $\begin{array}{l}\text { Construir perfiles psicográficos de profesores de educación básica de Arica } \\
\text { y establecer su relación con el SB. }\end{array}$ & DCF/AVR \\
\hline Saltijeral y Ramos, $2015^{77}$ & $\begin{array}{l}\text { México } \\
\text { Español }\end{array}$ & $\begin{array}{l}\text { Analizar la presencia y características del SB y las fuentes de estrés } \\
\text { percibidas por profesores de secundaria de México. }\end{array}$ & PCF \\
\hline Andaur y Berger, $2018^{78}$ & $\begin{array}{l}\text { Chile } \\
\text { Español }\end{array}$ & $\begin{array}{l}\text { Adaptar, implementar y medir el impacto de un taller de autocuidado } \\
\text { basado en mindfulness en los niveles de bienestar, satisfacción vital, estrés } \\
\text { y burnout en docentes y otros profesionales de la educación. }\end{array}$ & PI \\
\hline $\begin{array}{l}\text { Araujo, Morais, Faleiros y } \\
\text { Lobo, } 2016^{79}\end{array}$ & $\begin{array}{l}\text { Brasil } \\
\text { Portugués }\end{array}$ & $\begin{array}{l}\text { Levantar las producciones científicas que resaltan la importancia de } \\
\text { intervenciones grupales como estrategias para promover el } \\
\text { enfrentamiento y/o prevención de la enfermedad causada por el síndrome. }\end{array}$ & \multirow{4}{*}{ RL-EB } \\
\hline $\begin{array}{l}\text { Pereira, Ribeiro, Reis y } \\
\text { Fernandes, } 2018^{80}\end{array}$ & $\begin{array}{l}\text { Brasil } \\
\text { Portugués }\end{array}$ & $\begin{array}{l}\text { Sistematizar el conocimiento disponible en la literatura acerca del SB en } \\
\text { docentes actuantes en el curso de medicina. }\end{array}$ & \\
\hline $\begin{array}{l}\text { Ferreira, Silva, Bento, } \\
\text { Hernandez, Bernardo y } \\
\text { Silva, } 2015^{81}\end{array}$ & $\begin{array}{l}\text { Brasil } \\
\text { Portugués }\end{array}$ & $\begin{array}{l}\text { Analizar la literatura científica sobre las relaciones entre el SB y la } \\
\text { actividad física en profesores, a través } \\
\text { una revisión sistemática. }\end{array}$ & \\
\hline Andrade y Cardoso, $2012^{82}$ & $\begin{array}{l}\text { Brasil } \\
\text { Portugués }\end{array}$ & $\begin{array}{l}\text { Presentar algunas reflexiones acerca de los principales factores de riesgo } \\
\text { para el desarrollo del SB en los docentes, a fin de comprenderlos dentro de } \\
\text { un proceso de desgaste físico-emocional en el trabajo. }\end{array}$ & \\
\hline
\end{tabular}

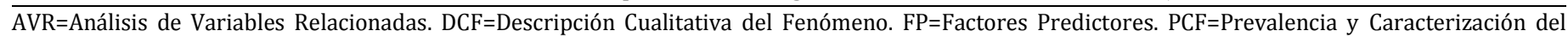
Fenómeno. PI=Programas de Intervención. RL-EB=Revisión de Literatura y/o Estudios Bibliométricos. VP=Validación de Pruebas.

Evaluar el SB en docentes que laboran medio y tiempo completo en dos universidades privadas.

Describir la prevalencia del SB entre los profesores de una escuela estatal de Brasil.

Comparar la presencia de indicadores de SB en tres grupos de docentes que actúan en el primer ciclo de enseñanza fundamental en Brasil. educativas privada y pública de Colombia enseñanza fundamental en $<$ Brasil. 2 entre profesores de educación especial y regular. universitarios brasileños. enseñanzas infantil, fundamental y media.

de primaria e investigar las posibles correlaciones entre el agotamiento, la depresión, demográficos y variables escuelas municipales de Brasil y su relación con variables

Batista, Carlotto, Coutinho Brasil

Brasil 
En ninguno de los países se reportaron investigaciones de los 4 tipos, no obstante, se destacó Brasil con la mayoría de estudios cuantitativos $(n=24)$, cualitativos $(n=3)$ y revisiones sistemáticas $(\mathrm{n}=4)$, siendo las de éste último tipo las únicas que se registraron en la presente investigación; por su parte, estudios mixtos se presentaron en Chile $(n=2)$, México (n=2) y Colombia (n=1) (Tabla 1).

Se evidenció que la publicación de artículos cuantitativos ha sido constante para el periodo de estudio, a diferencia de las investigaciones cualitativas, mixtas y revisiones sistemáticas, en las cuales no se realizaron publicaciones consecutivas. En cuanto al tipo de investigación según el idioma de publicación, predominó el portugués $(n=20)$ y español $(n=17)$ para los estudios cuantitativos, el portugués $(n=2)$ e inglés $(n=1)$ para los cualitativos, el español para los mixtos $(n=5)$ y el portugués para las revisiones sistemáticas $(\mathrm{n}=4)$.

Participantes de los estudios: 49 estudios mencionaron la muestra, de ellos 73,5\% se realizaron con docentes de instituciones de educación básica los demás con docentes de instituciones de educación superior. En cuanto al número de participantes de las investigaciones, 32 trabajaron con rangos inferiores a 200 individuos, 8 entre 201 a 400, 2 entre 401 a 600, 4 entre 601 a 800 y 3 entre 801 a 1000; de manera que las muestras más grandes fueron las reportadas en Brasil (Tabla 1).

Instrumentos y/o técnicas para la evaluación del SB: Los 41 a artículos cuantitativos aplicaron escalas, los estudios mixtos, señalaron el empleo de escalas junto a otras técnicas de recolección de información como: entrevistas en profundidad, entrevistas semiestructuradas, grupos focales, talleres y entrevistas en profundidad en conjunto con grupos focales ( $\mathrm{n}=1$ por cada categoría); en los estudios cualitativos, se identificó el uso de entrevistas semiestructuradas $(\mathrm{n}=2)$ y cuestionario de preguntas abiertas $(\mathrm{n}=1)$. En los 4 estudios de revisiones sistemáticas utilizaron matrices de registro.

Las escalas más usadas por las investigaciones cuantitativas y mixtas $(\mathrm{n}=46)$, fueron The Maslach Burnout Inventory (MBI) con 37\% y The Maslach Burnout Inventory para Educadores (MBI-Ed) con $15,2 \%$ las cuales evalúan las dimensiones de agotamiento emocional, despersonalización y realización personal; seguidamente, se ubicó el Cuestionario de Evaluación del Síndrome de
Quemarse por el Trabajo (CESQT) con $13 \%$ cuyas dimensiones son ilusión por el trabajo, desgaste psíquico, indolencia y culpa y por último el Cuestionario de Burnout para el Profesorado Revisado (CBP-R) con 8,7\%, en sus factores estrés y burnout, problemática administrativa $\mathrm{y}$ desorganización.

El $69,6 \%$ de los estudios dieron cuenta de propiedades psicométricas e indicaron valores de consistencia interna, dos estudios adicionalmente informaron de la validez de constructo por análisis factorial exploratorio (EFA) y 2 por análisis factorial confirmatorio (CFA), 1 refirió validez de constructo por EFA y validez de contenido por juicio de expertos, número semejante al que usó EFA y CFA simultáneos.

\section{Objetivos y principales resultados de los estudios} Los objetivos de los estudios se clasificaron en categorías de acuerdo a su similitud, a fin de identificar los alcances y frecuencia de los mismos, evidenciándose principalmente que el $66 \%$ se focalizaron en el análisis de variables relacionadas y en la determinación de la prevalencia y caracterización del SB (Tabla 1). Por su parte, los resultados de las investigaciones se estructuraron acorde con las siguientes categorías:

Análisis de variables relacionadas ( $n=25)$ : En cuanto a las variables individuales, se encontró que el sexo y la edad constituyen factores de riesgo que incrementan la probabilidad de que los docentes presenten el

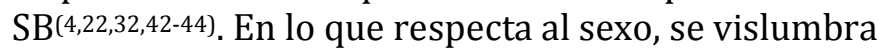
que las mujeres presentan mayores niveles de agotamiento emocional y realización personal (MBI)(4,22,42-44) e ilusión por el trabajo (CESQT) ${ }^{(32) ; ~ p o r ~}$ otro lado, se obsrevó que los hombres, tienen mayor despersonalización (MBI) $)^{(4,22,42-44)}$ y asimismo reportan niveles altos de indolencia y culpa (CESQT)(32).

En relación a la edad, un estudio reportó que los docentes de 51 a 60 años presentaron mayores niveles de realización personal $(\mathrm{MBI})^{(4)}$, en comparación con los otros grupos etarios evaluados. Otra investigación denotó que las personas menores de 45 años tienen mayor agotamiento emocional (MBI) frente a personas con edades mayores ${ }^{(44)}$. Cabe mencionar, que los estudios no indicaron asociaciones entre esta variable sociodemográfica y las demás dimensiones del MBI(4,44). 
Frente al estado civil, una investigación concluyó que los solteros presentan mayores niveles de agotamiento emocional y baja realización personal (MBI) ${ }^{(22)}$. En contraste otro estudio indicó que los docentes casados y divorciados se sienten más realizados a nivel personal (MBI) en comparación con aquellos que son separados o en unión libre(4). Estos estudios no denotaron relación entre estado civil y las otras dimensiones que evalúa el MBI. En torno al nivel de formación académica, se visualiza que los docentes con formación posgradual experimentan mayores niveles de realización personal y profesional (MBI) que los que tienen niveles educativos inferiores, no obstante, se vinculan a la presencia de mayores niveles de agotamiento emocional $(4,22,44)$.

En las variables laborales, una investigación señaló que a mayor antigüedad en la experiencia docente mayor realización personal y profesional y a menor antigüedad mayor despersonalización y agotamiento emocional(62). Así también, los elevados niveles de SB se asociaron a las variables condiciones inadecuadas de salud(54), ausentismo laboral(5), relaciones interpersonales desfavorables ${ }^{(47)}$ y percepción negativa de las condiciones del lugar de trabajo, las características y las demandas de la $\operatorname{tarea}^{(25,39,41,42,45,50,51,74,75) \text {. }}$

No se presentó una relación significativa entre el SB con el nivel de desempeño docente ${ }^{(53,55)}$ y la calidad de vida profesional(32); en contraposición, se vislumbró que elevados niveles de apoyo social, satisfacción laboral(31) y calidad de vida(49) son amortiguadores de las consecuencias negativas de dicho fenómeno.

Con relación a las variables psicológicas, un estudio señala que la ansiedad y depresión se asocian a mayores niveles de $\mathrm{SB}\left({ }^{48)}\right.$. Asimismo, se evidenció que los docentes con personalidad tipo inseguro presentan mayor indolencia y culpa y los de tipo empresario mayor ilusión por el trabajo(32). Por otra parte, se identificó que la autoeficacia(52), inteligencia emocional(39), valores normativos de tradición y obediencia $^{(46)}$ y uso de adecuadas estrategias de enfrentamiento( ${ }^{(40)}$ actúan como factores protectores del SB.

Prevalencia y caracterización del fenómeno $(n=10)$ : Se observó que la prevalencia y caracterización del SB, se ha estimado a través de diferentes instrumentos. En el caso de los estudios realizados en Brasil, que emplearon el MBI(60,61), las dimensiones de cansancio emocional y despersonalización se ubicaron en los niveles bajo y alto en el mayor porcentaje de la muestra y en la dimensión de realización personal en el nivel bajo(60,61).

Investigaciones que usaron el CESQT con profesores de educación especial, regular y universitaria, denotaron niveles altos en las dimensiones de desgaste psíquico, indolencia y culpa(64,65). Por otra parte, se obsrevaron niveles más altos en la dimensión "ilusión por el trabajo" en los docentes de enseñanza especial(64), en comparación al nivel obtenido por los profesores de educación regular(64) y universitaria(65).

Chavarría et al.(58) en su estudio sobre síndrome de burnout en médicos docentes de un hospital y el de Machado et al.(63) con profesores de la red pública en Brasil, aplicaron el CBP-R, encontrando una prevalencia de SB de $53,3 \%$ a $70,13 \%$ respectivamente.

El estudio de factores que prevalecen en el agotamiento profesional de los docentes, realizado en 2017 con el MBI-Ed(66), concluyó que el 59,3\% de los participantes se encontraba en la fase 2 de SB, etapa temprana de la enfermedad y el $24 \%$ en la fase 3 , la cual requiere de intervención.

Saltijeral et al.(77) en su estudio con trabajadores del Distrito Federal de México, utilizaron el CBP-R en conjunto con el MBI encontraron la existencia de puntajes moderados en las dimensiones de agotamiento emocional, baja realización personal, estrés de rol y percepción de falta de supervisión (CBP-R). Cabe señalar que si bien dentro de los instrumentos se alude el uso del MBI, no se presentan en el artículo los resultados derivados de su aplicación.

Por su parte, el estudio de Correa et al.(59), que usó del Cuestionario Breve de Burnout (CBB) reconoció una baja presencia de SB en los docentes, presentándose una frecuencia del 9\% de alta despersonalización, $16 \%$ altas consecuencias físicas y $9 \%$ altas consecuencias sociales. Por último, la investigación de Díaz et al.(62) que aplicó el Cuestionario de Burnout en Profesores Modificado (CBP-M) a docentes de colegios de la ciudad de Cali, evidenció bajos niveles de SB en todas las dimensiones, encontraron relación con el estrés de rol, supervisión, condiciones organizacionales y preocupaciones profesionales. 
Revisiones de literatura y/o estudios bibliométricos $(n=4)$ : Las investigaciones de este tipo, dan cuenta de que la investigación sobre el SB ha girado en torno a aspectos como prevalencia, estrategias de afrontamiento, factores de riesgo(79), factores desencadenantes ${ }^{(80)}$, conocimiento sobre el tópico(82) y efectos positivos de la actividad física o ejercicio sobre este fenómeno(81).

Prevalencia y caracterización del fenómeno y análisis de variables relacionadas $(n=3)$ : Se destaca que la prevalencia del SB evaluada a través del Spanish Burnout Inventory (SBI) osciló entre el 11\% a 40\%(67) $\mathrm{y}$ en el MBI-Ed entre el $0,7 \%$ a $43,4 \%$ en sus respectivas dimensiones(68,69). Conjuntamente las variables género femenino, no tener hijos, ser mayor de edad(69), presentar estabilidad en el trabajo, tener compañero fijo, considerar que la profesión es estresante( ${ }^{(68)}$ y presentar depresión( ${ }^{(67)}$ se asociaron significativamente con el SB.

Validación de pruebas (n=3): Los estudios de validación de pruebas sobre el SB se han desarrollado principalmente en lo que respecta a $\mathrm{MBI}\left({ }^{71)}\right.$ en Costa Rica, CESQT(72) en México y SBI(73) en Brasil, reportando adecuadas propiedades psicométricas.

Descripción cualitativa del fenómeno ( $n=3)$ : Dentro de las investigaciones orientadas a este objetivo, fue relevante la discriminación de los aspectos relacionales $\mathrm{y}$ organizacionales como factores desencadenantes del SB el significado, características del trabajo, expectativas realistas y apoyo social, como factores protectores (37). Asimismo, se resaltó la existencia de desconocimiento frente al $\mathrm{SB}^{(36)}$, y una predominancia del fenómeno en docentes de instituciones públicas( ${ }^{38)}$.

Factores predictores ( $n=2)$ : Se identificó como principales resultados en esta categoría que la autonomía, el apoyo social percibido(57) y dificultades con los estudiantes(56) actúan como factores predictores del SB, los cuales están inmersos en las esferas socio-laborales e interpersonales de los docentes(56,57).

Programas de intervención ( $n=2)$ : Los programas de intervención en $\mathrm{SB}$, se han centrado en auto diagnóstico, estrategias de enfrentamiento, gestión de problemas y emociones, gestión del tiempo/familia por trabajo, expectativas profesionales realísticas; este programa generó efectos positivos sobre el manejo del SB, caracterizándose por el desarrollo de sesiones que integraban un componente teórico, práctico y actividades de refuerzo para la casa(70); contrario a los resultados del programa de talleres de autocuidado basado en mindfulness( ${ }^{78)}$.

Descripción cualitativa del fenómeno y análisis de variables relacionadas $(n=1)$ : Este estudio mostró la existencia de cinco perfiles psicográficos en los docentes: indiferentes, realizados, dedicados temerosos, triviales y temerosos; los cuales se correlacionaron con el SB, destacándose el cansancio emocional y despersonalización en el perfil de docentes dedicados y temerosos y la realización personal en el perfil de realizados(76).

\section{Discusión}

El SB en la actualidad supone una de las afectaciones laborales de carácter psicosocial más importantes, que por la severidad de sus consecuencias ha propiciado mayor interés por parte de la comunidad científica nacional e internacional, así como de las entidades gubernamentales, estatales, empresariales y sindicales ${ }^{(2,22)}$.

La presente revisión sistemática evidencia que la investigación del SB en los países latinoamericanos muestra una tendencia creciente, especialmente entre 2014 a 2018, aspecto congruente a los hallazgos de otros estudios, los cuales concluyen que el interés manifiesto en las publicaciones sobre el tema ha ido en aumento en la última década, al ser considerado un fenómeno ocurrido en la interacción personatrabajo(12,30).

En cuanto al país de estudio, se destaca que las investigaciones sobre el tópico son mayoritariamente provenientes de Brasil, en contraposición a los resultados encontrados en otra revisión sistemática sobre SB, en donde México y Colombia representan el $50 \%$ de las investigaciones realizadas en Latinoamérica, aspecto que podría estar asociado a la población objeto de estudio, puesto que ésta no se hallaba limitada a docentes, al rango de años en que se realizó (2000 a 2010), las bases de datos consultadas (Science Direct, Scopus, Ebsco, Redalyc, Scielo y Google Académico) y el tipo de estudios incorporados (publicaciones en revistas científicas, libros y memorias de eventos)(12).

En relación a los alcances metodológicos, se identifica en esta revisión el desarrollo de investigaciones 
preponderantemente cuantitativas, de carácter descriptivo, correlacional y diseño transversal (Tabla 1), denotando la existencia limitada de estudios de naturaleza experimental o interventivo, que incluyan diseños longitudinales o se orienten a la comprensión cualitativa del SB. Lo expuesto, es coherente con las conclusiones arrojadas por otras revisiones sistemáticas sobre SB, las cuales evidencian hallazgos semejantes y explicitan la necesidad de enriquecer los ejercicios investigativos con diversos paradigmas metodológicos, a fin de realizar un abordaje más integral del fenómeno(11,30).

Frente a los participantes de los estudios, se reconoce que las investigaciones sobre el SB en el área de docencia son más frecuentes en los profesionales de instituciones de educación básica ${ }^{(24,33,34)}$. Si bien otros estudios sobre el tema no presentan una comparación en términos de publicación científica dependiendo de la etapa educativa en la que los docentes imparten sus cátedras, éstos coinciden en sostener que el sector educativo es uno de los más vulnerables a presentar el SB(5), siendo necesario dar continuidad a las investigaciones sobre el tópico para poder actuar de manera oportuna ${ }^{(24,33,34)}$.

Estudios indican que la investigación sobre el SB en Latinoamérica presenta una propensión a mantener los lineamientos de países norteamericanos y europeos a nivel paradigmático y metodológico, hecho evidente en la notoria recurrencia a emplear el MBI como instrumento de medición de este fenómeno(3,11,12). Todo ello, se hace manifiesto en los estudios incorporados en ésta revisión sistemática, en los que se hace uso frecuentemente de la versión original del mismo (MBI) y en menor medida de su versión para población docente (MBI-Ed), en países como Brasil(46,66), Colombia(22), México(48,77), y Chile(5) principalmente.

Los objetivos de los estudios considerados en esta revisión privilegian el análisis de las variables relacionadas al SB, seguidos de la determinación de su prevalencia. De esta manera, atendiendo a las primeras investigaciones, los principales hallazgos denotan la asociación con características sociodemográficas, laborales y psicológicas de los docentes (Tabla 1).

Dentro de las variables sociodemográficas, se manifiesta que en las mujeres y personas jóvenes hay mayor tendencia a desarrollar el SB; en este sentido, frente al sexo otros estudios aportan conclusiones semejantes al exponer que las docentes mujeres constituyen un grupo vulnerable a presentar el síndrome, dado el cumplimiento de demandas tanto laborales como del hogar y la sobrecarga que éstas representan(12,23). De manera similar, en lo que alude a la edad algunos estudios indican el alcance de puntuaciones más elevadas en las dimensiones del SB en los docentes jóvenes, debido a la incongruencia entre las expectativas idealistas establecidas y la realidad laboral cotidiana ${ }^{(23,33) .}$.

Por otro lado, los hallazgos de esta revisión indican que las personas solteras y con altos niveles de formación académica tienen una propensión mayor a presentar SB. Lo planteado, es equiparable a los resultados de otras investigaciones, las cuales concluyen que en las personas solteras existen menores redes de apoyo social y en aquellos profesionales con formación de doctorado, las exigencias laborales son más altas en comparación a individuos con niveles de estudios inferiores(34). En contraste, existen investigaciones que denotan que las variables sociodemográficas señaladas anteriormente no muestran ser factores importantes en relación a la presencia del $\mathrm{SB}^{(29,33,34)}$.

Frente a las variables laborales, los resultados de los estudios incluidos en la pesquisa reportan que la percepción de apoyo social y satisfacción laboral, pueden ser considerados factores protectores frente al SB, elemento coherente a los datos de otras investigaciones, los cuales afirman que éstos actúan como amortiguadores de sus consecuencias negativas(11,23,33).

Así también, existe asociación positiva entre algunas dimensiones del SB con las variables antigüedad ${ }^{43} y$ ausentismo laboral(5), condiciones inadecuadas de salud y percepción negativa de las condiciones del lugar de trabajo(48,55,67), características, demandas de la tarea y relaciones interpersonales $(4,5,22,31,32,41$ $45,47,49,74)$. Los aspectos enunciados son semejantes a los derivados de otros estudios, debido a que coinciden en postular que dichos factores cumplen la función de antecedentes en el desarrollo del $\mathrm{SB}(11,22,33)$, a excepción de un estudio que concluye que la valoración de las condiciones del trabajo como buenas o malas y el juicio de la dificultad de la tarea laboral, no muestra ser un indicador importante de relación con el síndrome(29).

En cuanto a las variables psicológicas, se destacan la ansiedad, depresión y tipos de personalidad como 
factores relacionados al SB, elementos reforzados por los resultados de otras investigaciones que presentan los mismos hallazgos y sus efectos negativos sobre la calidad de vida laboral(1,29). Del mismo modo, se entrevé asociación de dicho fenómeno con la autoeficacia e inteligencia emocional, valores normativos de tradición y obediencia y uso adecuado de estrategias de enfrentamiento, elementos catalogados por estudios semejantes como factores mediadores del SB, atribuibles a las diferencias individuales en la adaptación de las personas al síndrome ${ }^{(23,29) .}$

Finalmente, en lo que concierne a las investigaciones que tuvieron como objetivo determinar la prevalencia del SB en los docentes, se resalta que su estimación se ha realizado a través de diversos instrumentos de medición, instalados en posturas teóricas disímiles, que han generado mediciones muy distintas las unas de las otras. Asimismo, los resultados arrojados en dichos estudios, muestran gran heterogeneidad en los criterios de determinación de los niveles o presencia del SB, cuyas interpretaciones se han delimitado por medio de frecuencias, porcentajes, medias y medianas y/o categorías o niveles de Burnout, dificultando la comparación de resultados.

Dichos elementos, se contrastan con los resultados de otras investigaciones sobre el tema, puesto que presuponen la existencia de dificultades en torno al establecimiento de la prevalencia poblacional del fenómeno, generando sobreestimación o subestimación de la misma y ocasionando una parcialización de sus conclusiones e impedimentos para la comprensión de relaciones causales con otras variables, la forma de abordaje de cada una de ellas, y por tanto, su tratamiento en beneficio de los sujetos que presentan SB, incluyendo estrategias orientadas a nivel individual (fisiológicas, cognitivas y conductuales), organizacional y social(11,30,83).

\section{Conclusiones}

Las investigaciones desarrolladas sobre el SB durante los últimos años, se han concentrado en algunos países latinoamericanos, en ellas la mayoría de participantes corresponden a docentes de instituciones de educación básica y media, aspecto coherente con la idea de que estos grupos son altamente vulnerables a presentar dicho fenómeno, denotando la necesidad de desarrollar más estudios centrados en docentes de educación universitaria.
Los estudios revisados emplearon metodologías predominantemente cuantitativas, siendo el Maslach Burnout Inventory (MBI) el instrumento de recolección de información más usado. A futuro es importante realizar estudios primarios sobre dicho fenómeno, los cuales opten por diseños metodológicos diversos que profundicen la comprensión del fenómeno en docentes, indaguen sobre las posibles diferencias en su prevalencia, en función de las características de los grupos poblacionales y el tipo de instrumento utilizado.

En relación a los objetivos dispuestos en los estudios analizados, muestran que estos se han orientado en mayor frecuencia al análisis de variables relacionadas al fenómeno, seguidos por la identificación de la prevalencia y caracterización del mismo. De aquellos estudios que se centraron en el primer objetivo, se concluye la existencia de relaciones entre el SB y variables de carácter sociodemográfico, laboral y psicológico, las cuales pueden actuar como potenciales factores de riesgo o protección en el desarrollo del síndrome. Las investigaciones enfocadas en el segundo objetivo, arrojan como principales hallazgos la heterogeneidad presente en los instrumentos y criterios para estimar la prevalencia, con niveles variables de SB, aspecto que señala la significancia de efectuar estudios que apunten a la estructuración de puntos de corte, con base en criterios unificados y acordes a la población docente latinoamericana.

Estos resultados explicitan la necesidad de diseñar y desarrollar investigaciones que reporten resultados de procesos de intervención para el SB, considerando a su vez programas de promoción y prevención, indispensables en el contexto del trabajo, puesto que la revisión señala la existencia de limitada producción científica en este ámbito.

\section{Limitaciones}

Para la realización de próximos estudios, se recomienda delimitar mejor el tema debido a la extensión del mismo, con el fin de ampliar su comprensión al análisis de desarrollos conceptuales y empíricos del SB. Así también, es prudente señalar la cantidad y generalidad de las bases de datos consultadas, dado que dichos aspectos probablemente redujeron el cúmulo de artículos a los cuales se accedió.

Finalmente, se precisa relevante exponer como fortaleza importante de la presente investigación el 
hecho de conjugar en su objetivo variables que habitualmente suelen separarse en las revisiones sistemáticas, de tal forma que su contenido resulta transcendental a nivel latinoamericano.

Conflicto de intereses: Los autores declaran que no existen conflictos de intereses.

\section{Referencias}

1. Ojeda G. Modelo causal social cognitivo de burnout en profesionales de la salud de Lima Metropolitana [Internet]. Tesis digitales - UNMSM. Universidad Nacional Mayor de San Marcos; 2016. Disponible en: http://cybertesis.unmsm.edu.pe/handle/cybertesis/4930

2. Organización Mundial de la Salud. Efectos del empelo precario en la salud y la seguridad en el trabajo [Internet]. 2002 [cited 2019 Mar 13]. Disponible en: https://www.who.int/occupational_health/publications/ne wsletter/en/gohnet2s.pdf

3. Patlán Pérez J. Efecto del burnout y la sobrecarga en la calidad de vida en el trabajo. Estudios Gerenciales [Internet]. 2013 [cited 2019 Mar 13];29:445-55. Disponible en: http://crossmark.crossref.org/dialog/?doi=10.1016/j.estge rwww.elsevier.es/estudios_gerenciales

4. Rodríguez EA, Sánchez M de los Á. Síndrome de Burnout y variables sociodemográficas en docentes de una universidad privada de Lima. Revista de Investigación Educativa. 2018;36(2):401-19. doi.org/10.6018/rie.36.2.282661

5. Jorquera R, Orellana C, Tapia C, Vergara E. Síndrome de Burnout en una muestra de profesores / as de enseñanza básica de la ciudad de Copiapó. Summa Psicológica UST. 2014;11(2):115-34. disponible en: https://dialnet.unirioja.es/servlet/articulo?codigo=495399 4

6. Freudenberger HJ. Staff Burn-Out. Journal of Social Issues [Internet]. 1974 Jan [cited 2020 Jan 11];30(1):159-65. https://doi.org/10.1111/j.1540-4560.1974.tb00706.x

7. Maslach C, Jackson SE. The measurement of experienced burnout. Journal Of Occupational Behaviour [Internet]. 1981 [cited 2019 Mar 13];2(2):99-113. https://doi.org/10.1002/job.4030020205

8. Gil-Monte P. El síndrome de quemarse por el trabajo (burnout). Una enfermedad laboral en la sociedad del bienestar. [Internet]. Pirámide. Madrid, España; 2005 [cited 2020 Jan 11]. 3879-3882 p. Disponible en: http://scielo.isciii.es/pdf/neuropsiq/n94/v25n2a09.pdf

9. Gil-Monte P. El síndrome de quemarse por el trabajo (burnout): una perspectiva histórica. In: Quemarse en el trabajo: 11 perspectivas del burnout [Internet]. Egido Edit. Madrid, España: P. Gil-Monte y B. Moreno-Jiménez (Comps.); 2007 [cited 2020 Jan 11]. p. 37-71. Disponible en: https://www.researchgate.net/publication/263278349_El_ sindrome_de_quemarse_por_el_trabajo_burnout_una_perspe ctiva_historica

10. Martínez Pérez A. El Síndrome de Burnout. Evolución conceptual y estado actual de la cuestión. Vivat Academia [Internet]. 2010;(112):42-80. https://doi.org/10.15178/va.2010.112.42-80

11. Salazar Becerril A. Salud, burnout y estrés en ámbitos laborales. Una revisión sistemática. Psicologia y Salud [Internet]. 2015;25(2):147-55. Disponible en: http://mastor.cl/blog/wp-

content/uploads/2018/02/Becerril-2015.-Salud-bernout-yestres.-pdf.pdf

12. Díaz Bambula F, Carolina Gómez I. La investigación sobre el síndrome de burnout en latinoamérica entre 2000 y el 2010. Psicología desde el Caribe [Internet]. 2016 [cited 2019 Mar 13];33(1):113-31. Disponible en: https://doi.org/10.14482/psdc.33.1.8065

13. Harrison W. A social competence model of burnout. In: Farber B, editor. Stress and burnout in the human services professions. Pergamon P. New York, EEUU; 1983. p. 29-39.

14. Pines AM. Burnout: An existential perspective. In: Schaufeli WB, Maslach C, Marek T, editors. Series in applied psychology: Social issues and questions Professional burnout: Recent developments in theory and research [Internet]. Taylor \& Francis; 1993 [cited 2020 Jan 12]. p. 3351. Disponible en: https://www.taylorfrancis.com/books/e/9781315227979/ chapters/10.4324/9781315227979-4

15. Cherniss C. Staff Burnout: Job Stress in the Human Services (Sage studies in community mental health). Beberly Hills: SAGE Publications, Inc; 1980. 197 p. https://books.google.com.co/books/about/Staff_Burnout.ht ml?id=XVZqAAAAMAAJ\&redir_esc $=y$

16. Buunk BP, Schaufeli WB, Ybema JF. Burnout, Uncertainty, and the Desire for Social Comparison Among Nurses. Journal of Applied Social Psychology. 1994;24(19):1701-18. https://doi.org/10.1111/j.1559-1816.1994.tb01570.x

17. Hobfoll S, Freedy J. Conservation of Resources: A General Stress Theory Applied To Burnout. In: Schaufeli WB, Maslach C, Marek T, editors. Professional burnout: Recent developments in theory and research [Internet]. Taylor \& F. London; 1993 [cited 2020 Jan 11]. p. 115-29. Disponible en: https://www.taylorfrancis.com/books/e/9781315227979/ chapters/10.4324/9781315227979-4

18. Golembiewski RT, Boudreau $\mathrm{R}$, Goto $\mathrm{K}$, Murai $\mathrm{T}$. Transnational perspective on job burnout: Replication of phase model results among japanese respondents. The International Journal of Organizational Analysis. 1993;1(1):7-27. https://doi.org/10.1108/eb028781

19. Cox T, Kuk G, Leiter MP. Burnout, health, work stress, and organizational healthiness. In: Schaufeli WB, Maslach C, Marek T, editors. Professional burnout: Recent developments in theory and research [Internet]. Taylor \& F. Londres; 1993 [cited 2020 Jan 11]. p. 177-93. Disponible en: https://psycnet.apa.org/record/1993-97794-011

20. Winnubst J. Organizational structure, social support, and burnout. In: Schaufeli WB, Maslach C, Marek. T, editors. Professional burnout: Recent developments in theory and research [Internet]. Londres: Taylor and Francis; 1993 [cited 2020 Jan 12]. p. 151-62. Disponible en: https://www.taylorfrancis.com/books/e/9781315227979/ chapters/10.4324/9781315227979-12

21. Gil-Monte P, Peiró J. Un estudio comparativo sobre criterios normativos y diferenciales para el diagnóstico del síndrome de quemarse por el trabajo (burnout) según el MBI-HSS en España. Psicología del Trabajo y de las Organizaciones [Internet]. 2000 [cited 2020 Jan 11];2:135-49. Disponible en:

https://www.researchgate.net/publication/263232651_Un _estudio_comparativo_sobre_criterios_normativos_y_diferen ciales_para_el_diagnostico_del_sindrome_de_quemarse_por_ el_trabajo_burnout_segun_el_MBI-HSS_en_Espana 
22. Marenco AD, Ávila JH. Burnout y problemas de salud mental en docentes: diferencias según características demográficas y sociolaborales. Psychologia: Avances de la Disciplina [Internet]. 2016 [cited 2019 Mar 13];10(1):91-100. Disponible en: http://www.scielo.org.co/pdf/psych/v10n1/v10n1a09.pdf

23. Ramírez M, Zurita R. Variables organizacionales $y$ psicosociales asociadas al Síndrome de Burnout en trabajadores del ámbito educacional. Polis. 2010;9(25):51534. https://doi.org/10.4067/s0718-65682010000100029

24. Botero Álvarez C. Riesgo psicosocial intralaboral y "burnout" en docentes universitarios de algunos países latinoamericanos. Cuadernos de Administración. 2012;28(48):117-32. Disponible en: http://www.scielo.org.co/pdf/cuadm/v28n48/v28n48a10. pdf

25. Silva NR, Bolsoni A, Rodrigues OM, Capellini VL. O trabalho do professor, indicadores de Burnout, práticas educativas e comportamento dos alunos: Correlação e Predição. Revista Brasileira de Educação Especial. 2015;21(3):363-76. https://doi.org/http://dx.doi.org/10.1590/S141365382115000300004

26. Castillo D, Alzamora M. Síndrome de Burnout en docentes que se desempeñan en escuelas públicas vulnerables de la ciudad de Santiago. AKADÉMEIA. 2015;6(1):28-41. Disponible

https://www.semanticscholar.org/paper/S\%C3\%ADndrom e-de-Burnout-en-docentes-que-se-desempe\%C3\%B1an-

Castillo-

Alzamora/fcad9f8f154ab01f0268c3326f8c38aeb31c1b85

27. Bustamante E, Bustamante F, González G, Bustamante L. El burnout en la profesión docente: un estudio en la escuela de bioanálisis de la Universidad de Carabobo Sede Aragua, Venezuela. Medicina y Seguridad del Trabajo [Internet]. 2016 [cited 2019 Mar 13];62(243):111-21. Disponible en: http://scielo.isciii.es/scielo.php?script=sci_arttext\&pid=S04 65-546X2016000200003\&lng=es\&tlng=es

28. Enríquez MF, Velásquez F. El burnout en la práctica pedagógica del docente del docente de San Juan de Pasto. Universidad y Salud [Internet]. 2012 [cited 2019 Mar 13];1(15):50-66. Disponible en: http://revistas.udenar.edu.co/index.php/usalud/article/vie w/1267/pdf_7

29. Ávila J, Gómez L, Montiel M. Características demográficas y laborales asociadas al Síndrome de Burnout en profesionales de la salud. Pensamiento Psicologico. 2010;8(15):39-51. Disponible https://www.redalyc.org/pdf/801/80115648004.pdf

30. Juárez-García A, Idrovo A, Camacho-Ávila A, Placencia-Reyes O. Síndrome de burnout en población mexicana: Una revisión sistemática. Salud Mental [Internet]. 2014 [cited 2019 Mar 13];37(2):159-76. Disponible en: http://www.redalyc.org/articulo.oa?id=58231307010

31. Jiménez A, Jara MJ, Miranda E. Burnout, apoyo social y satisfacción laboral en docentes. Psicologia Escolar e Educacional. 2012;16(1):125-34. https://doi.org/10.1590/s1413-85572012000100013

32. Salgado JA, Leria FJ. Síndrome de burnout y calidad de vida profesional percibida según estilos de personalidad en profesores de educación primaria. CES Psicología. 2018;11(1):69-89. Disponible en: https://revistas.ces.edu.co/index.php/psicologia/article/vi ew/4192
33. Olaya Arévalo C. Síndrome de Burnout o síndrome de agotamiento profesional (SAP) en el trabajo de los docentes distritales de la localidad de Usme [Internet]. Universidad Militar Nueva Granada; 2015 [cited 2020 Jan 12]. Disponible en:

https://repository.unimilitar.edu.co/handle/10654/6691

34. Palmer Morales L, Prince Velez R, Medina Ramirez C, Figueroa Largada M, López Martinez R, Rodriguez Rodriguez G. Prevalencia del síndrome de Burnout en docentes de la Universidad autónoma de Baja California, Mexicali, México. Revista Cubana de salud y Trabajo [Internet]. 2016;17(3):36-40. Disponible en: http://www.medigraphic.com/pdfs/revcubsaltra/cst2016/cst163g.pdf

35. García Peñalvo JF. Revisión sistemática de literatura en los Trabajos de Final de Máster y en las Tesis Doctorales. Universidad de Salamanca; 2017. https://doi.org/10.5281/zenodo.399302

36. Diehl L, Carlotto MS. Knowledge of teachers about the Burnout Syndrome: process, risk factors and consequences. Psicologia em Estudo [Internet]. 2014;19(4):741-52. https://doi.org/10.1590/1413-73722455415

37. Gomes Teixeira F, Santos da Silva MR, Medeiros GL. Síndrome de Burnout - a interface entre o trabalho na área da educação e na enfermagem. Revista de Enfermagem Referência. 2010;3(2):101-9.

http://www.scielo.mec.pt/pdf/ref/vserIIIn2/serIIIn2a11.p df

38. Esteves Ferreira AA, Santos DE, Rigolon RG. Avaliação comparativa dos sintomas da síndrome de burnout em professores de escolas públicas e privadas. Revista Brasileira de Educacao. 2014;19(59):987-1002. https://doi.org/10.1590/S1413-24782014000900009

39. Ilaja B, Reyes C. Burnout y estrategias de inteligencia emocional en profesores universitarios: implicaciones en la salud laboral educativa. Psicología desde el Caribe [Internet]. 2016;33(1):31-46. https://doi.org/10.14482/psdc.33.1.8081

40. Mazon V, Carlotto MS, Câmara S. Síndrome de Burnout e estratégias de enfrentamento em professores. Arquivos Brasileiros de Psicologia [Internet]. 2008;60(1):55-66. Disponible

en: http://www.redalyc.org/articulo.oa?id=229017544006\%0 AComo

41. Costa BE, Silva NLS. Analysis of environmental factors affecting the quality of teacher's life of public schools from Umuarama. Work. 2012;41:3693-700. https://doi.org/10.3233/WOR-2012-0673-3693

42. Santana ÂM, De Marchi D, Junior L, Girondoli Y, Chiappeta A. Burnout syndrome, working conditions, and health: A reality among public high school teachers in Brazil. Work. 2012;41:3709-17. https://doi.org/10.3233/WOR-20120674-3709

43. Bedoya E, Vega N, Severiche C, Meza M. Síndrome de Quemado (Burnout) en docentes universitarios: el caso de un centro de estudios del caribe colombiano. Formación Universitaria [Internet]. 2017;10(6):51-8. Disponible en: http://dx.doi.org/10.4067/S0718-50062017000600006

44. Ruiz D, Pando M, Aranda C, Almeida C. Burnout y Work Engagement en docentes universitarios de Zacatecas. Ciencia \& Trabajo. 2014;15(50):116-20. https://doi.org/10.4067/S0718-24492014000200010

45. Moreno B, Corso S, Sanz AI, Rodriguez A, Boada M. El "Burnout" y el "Engagement" en profesores de Perú. 
Aplicación del modelo de demandas-recursos laborales. Ansiedad y Estrés. 2010;16(2-3):293-307. Disponible en: https://pesquisa.bvsalud.org/portal/resource/\%20es/ibc91868

46. Souza S, Tenório F, Cruz SI, Sousa R, Gomes D. Síndrome de burnout e valores humanos em professores da rede pública estadual da cidade de João Pessoa: Um estudo correlacional. Analise Psicologica. 2016;34(2):119-31. https://doi.org/10.14417/ap.910

47. Bruch L, Aerts D, Guimarães G, Gonçalves S. The Burnout Syndrome in professors of private higher education institutions of Santarém , PA. Tempus. 2016;10(3):115-36. https://doi.org/DOI:

http://dx.doi.org/10.18569/tempus.v10i3.1872 A

48. Sánchez F, Velasco JJ. Comorbilidad entre síndrome de burnout, depresión y ansiedad en una muestra de profesores de educación básica del Estado de México. Papeles de Poblacion. 2017;23(94):261-86. https://doi.org/10.22185/24487147.2017.94.038

49. Tabeleão V, Tomasi E, Neves S. Qualidade de vida e esgotamento profissional entre docentes da rede pública de Ensino Médio e Fundamental no Sul do Brasil. Cad Saúde Pública. 2011;27(12):2401-8. https://doi.org/10.1590/S0102-311X2011001200011

50. Carlotto MS, Cámara SG. Burnout Syndrome Profiles among Teachers. Escritos de Psicología / Psychological Writings. 2017;10(3):159-66. https://doi.org/10.5231/psy.writ.2017.2911

51. Camargo G, Nesello F, Santos H, Lopes F, Durán A, Eumann A, et al. Fatores associados a piores níveis na escala de Burnout em professores da educação básica. Cadernos Saúde Coletiva. 2015;23(3):268-75. https://doi.org/10.1590/1414$462 \times 201500030121$

52. Carlotto MS, Silva SR, Vidal JB, Diehl L. O papel mediador da autoeficácia na relação entre a sobrecarga de trabalho e as dimensões de Burnout em professores. Psico-USF. 2015;20(1):13-23. https://doi.org/10.1590/141382712015200102

53. García A, Escorcia CV, Perez BS. Síndrome de Burnout y sentimiento de autoeficacia en profesores universitarios. Propositos y Representaciones [Internet]. 2017;5(2):65-96. https://doi.org/http://dx.doi.org/10.20511/pyr2017.v5n2. 170

54. Suda Y, Coelho A, Bertaci A, Santos B. Relação entre nível geral de saúde, dor musculoesquelética e síndrome de burnout em professores universitários. Fisioterapia e Pesquisa,. 2011;18(3):270-4. https://doi.org/10.1590/S1809-29502011000300012

55. Cárdenas M, Méndez L, González M. Evaluación del desempeño docente, estrés y Burnout en profesores universitarios. Revista Electrónica Actualidades Investigativas en Educación [Internet]. 2014;14(1):1-22. http://www.redalyc.org/articulo.oa?id=44729876006\%0A Cómo

56. Dalagasperina P, Monteiro JK. Preditores da síndrome de burnout em docentes do ensino privado. Psico-USF. 2014;19(2):265-75. https://doi.org/10.1590/141382712014019002011

57. Carlotto M, Câmara S. Riscos psicossociais associados à síndrome de burnout em professores universitários. Avances en Psicología Latinoamericana. 2017;35(3):447-57. Disponible en: http://www.scielo.org.co/scielo.php?script=sci_abstract $\&$ pi $\mathrm{d}=\mathrm{S} 1794-47242017000300447$
58. Chavarría RA, Colunga FJ, Loria J, Peláez K. Síndrome de burnout en médicos docentes de un hospital de $2 .^{\circ}$ nivel en México. Educacion Medica [Internet]. 2017;18(4):254-61. Disponible http://dx.doi.org/10.1016/j.edumed.2016.09.001

59. Correa Z, Muñoz I, Chaparro A. Síndrome de Burnout en docentes de dos universidades de Popayán, Colombia. Revista de Salud Pública. 2010;12(4):589-98. https://doi.org/10.1590/s0124-00642010000400006

60. Lima JL, Cardoso L, Pereira M, Alves PA, Gomes T, Amaral E. Prevalência da síndrome de Burnout entre professores da Escola Estadual em Niterói, Brasil. Revista Enfermería Actual. 2018;18(34):14-25 https://doi.org/http://dx.doi.org/10.15517/revenf.v0i34.3 0262

61. Silva N, Almeida MA. As características dos alunos são determinantes para o adoecimento de professores- um estudo comparativo sobre a incidência de Burnout em professores do ensino regular e especial. Rev Bras Ed Esp. 2011;17(3):373-94. https://doi.org/10.1590/S141365382011000300003

62. Díaz F, López AM, Varela MT. Factores asociados al síndrome de burnout en docentes de colegios de la ciudad de Cali, Colombia. Universitas Psychologica. 2012;11(1):217-27. https://doi.org/10.11144/Javeriana.upsy11-1.fasb

63. Machado GC, Nunes F de P, Absalão CA. Síndrome de Burnout em professores da rede pública. Production. 2009;19(3):458-65. https://doi.org/10.1590/s010365132009000300004

64. Braun AC, Carlotto MS. Síndrome de Burnout: Estudo comparativo entre professores do ensino especial e do ensino regular. Psicologia Escolar e Educacional. 2014;18(1):125-33. https://doi.org/10.1590/S141385572014000100013

65. Costa L, Possobon R de F, Ambrosano GM, Gil-Monte PR. Prevalence of burnout syndrome in a sample of brazilian university professors. Psicologia: Reflexao e Critica. 2013;26(4):636-42. https://doi.org/10.1590/S010279722013000400003

66. Silva A, Maia M de F, Lima CA, Guedes I, Pedreira K, Silva DA, et al. Fatores Que Prevalecem Ao Esgotamento Profissional Em Professores. Cadernos Brasileiros de Terapia Ocupacional. https://doi.org/10.4322/0104-4931.ctoao0822

67. Silva NR, Bolsoni AT, Loureiro SR. Burnout e depressão em professores do ensino fundamental: um estudo correlacional. Revista Brasileira de Educação. 2018;23:1-18. https://doi.org/10.1590/s1413-24782018230048

68. Batista JB, Carlotto MS, Coutinho AS, Augusto LG. Prevalência da Síndrome de Burnout e fatores sociodemográficos e laborais em professores de escolas municipais da cidade de João Pessoa , PB. Rev Bras Epidemiol. 2010;13(3):502-12. https://doi.org/10.1590/S1415-790X2010000300013

69. Carlotto MS. Síndrome de Burnout em Professores: Prevalência e Fatores Associados. Psicologia: Teoria e Pesquisa. 2011;27(4):403-10. http://dx.doi.org/10.1590/S0102-37722011000400003

70. Dalcin L, Carlotto MS. Avaliação de efeito de uma intervenção para a Síndrome de Burnout em professores. Psicologia Escolar e Educacional. 2018;22(1):141-50. https://doi.org/10.1590/2175-35392018013718

71. Alvarado K. Validez factorial de Maslach Burnout Inventor (Versión castellana) en educadores costarrienses. 
Actualidades Investigativas en Educación. 2009;9(1):1-22. https://revistas.ucr.ac.cr/index.php/aie/article/view/9385

72. Gil-Monte PR, Unda S, Sandoval J. Validez factorial del Cuestionario para la Evaluación del Síndrome de Quemarse por el Trabajo (CESQT) en una muestra de maestros mexicanos. Salud mental. 2009;32(3):205-14. Disponible en: https://www.redalyc.org/pdf/582/58212279004.pdf

73. Gil-monte P, Carlotto MS, Câmara S. Validation of the Brazilian version of the "Spanish Burnout Inventory" in teachers. Rev Saúde Pública. 2010;44(1):1-8. https://doi.org/10.1590/S0034-89102010000100015

74. Preciado MDL, Pozos E, Colunga C, Vázquez JM, Ávalos ML. Relación entre factores psicosociales, agotamiento emocional laboral y burnout en odontólogos mexicanos. Universitas Psychologica. 2017;16(2):1-11. https://doi.org/10.11144/javeriana.upsy16-2.refp

75. Muñoz-Muñoz CF, Correa-Otalvaro CM. El papel del docente reflexivo como estrategia del síndrome de Burnout en relación con las prácticas educativas. Hallazgos. 2014;11(22):385-401. https://doi.org/10.15332/s17943841.2014.0022.19

76. Cuadra A, Lee S, Sossa P. Construcción de perfiles psicográficos de profesores de educacion basica de escuelas municipales de Arica. Estudios pedagógicos. 2009;35(1):1326. https://doi.org/10.4067/s0718-07052009000100001

77. Saltijeral MT, Ramos L. Identificación de estresores laborales y burnout en docentes de una secundaria para trabajadores del Distrito Federal. Salud Mental. 2015;38(5):361-9. https://doi.org/10.17711/SM.0185-3325.2015.049

78. Andaur A, Berger C. Implementación e impacto de un taller de autocuidado basado en mindfulness en profesionales de la educación. Estudios Sobre Educacion. 2018;34:239-61. https://doi.org/10.15581/004.34.239-261

79. Araújo L De, Morais V, Faleiros A, Lobo CM. Intervenção grupal com professores com síndrome de burnout: uma revisão integrativa. Revista de Epidemiologia e Controle de Infecção. 2016;1(1):203-13. https://doi.org/https://doi.org/10.17058/reci.v1i1.7977

80. Pereira C, Ribeiro LA, Reis E, Fernandes P. Síndrome de Burnout em professores de medicina: revisão sistemática. Revista EDaPECI. 2018;18(3):115-26. Disponible en: https://seer.ufs.br/index.php/edapeci/article/view/10026

81. Ferreira E, Silva F, Bento G, Hernandez S, Bernardo V, Silva R. Revisão sistemática sobre Síndrome de Burnout e atividade fisica em profesores. Educación Física y Deporte. 2015;34(2):309-30.

https://doi.org/10.17533/udea.efyd.v34n2a02

82. Andrade P, Cardoso T. Prazer e dor na docência: revisâo bibliográfica sobre a Síndrome de Burnout. Saúde Soc São Paulo. 2012;21(1):129-40. https://doi.org/10.1590/S010412902012000100013

83. Domínguez E. Burnout, estrategias de afrontamiento y prevención en trabajadores sociales [Internet]. Universidad de la Rioja; 2015. Disponible en: https://biblioteca.unirioja.es/tfe_e/TFE000978.pdf?fbclid=I wAR0q11gN3-

ajXJM0DEpV6rjMUrRQrx6v3dyoM91vf7D8N8r86EYBkHvV yJg 